\title{
Ácaros asociados al coleóptero Passalus cognatus (Coleoptera: Passalidae) de Los Tuxtlas, Veracruz, México
}

\author{
Gabriel A. Villegas-Guzmán ${ }^{1}$, Tila M. Pérez ${ }^{1} \&$ Pedro Reyes-Castillo ${ }^{2}$ \\ 1. Colección Nacional de Ácaros, Departamento Zoología, Instituto de Biología, Universidad Nacional Autónoma de \\ México. A.P. 70-153, Ciudad Universitaria, 04510 México, D. F. Tel.: (55) 5622 9142, Fax (55) 5550 0164; gabrvill@ \\ yahoo.com, tilam@ibiologia.unam.mx, \\ 2. Instituto de Ecología, A. C. Apartado Postal 63, 91000 Xalapa, Veracruz, México; pedro.reyes@inecol.edu.mx
}

Recibido 11-VI-2007. Corregido 21-IX-2007. Aceptado 31-VII-2008.

\begin{abstract}
Mites associated to the Coleopteran Passalus cognatus (Coleoptera:Passalidae) from Los Tuxtlas, Veracruz, Mexico. There are few records of mites associated with the tropical coleopterans of Mexico. We examined 35 passalid beetles (bessbugs) Passalus cognatus from Los Tuxtlas region in Veracruz State, Mexico. Twenty of them had a total of 245 mites (representing eight species, eight genera, eight families and three suborders). The most abundant species were Uroobovella californiana Wisniewski \& Hirschmann (35\%), Euzercon hyatti Hunter \& Rosario (20\%), and Uropoda sp. (17.5\%). The preferred attachment areas were the coxae; followed by the mesosternum and the humeri. Each beetle had 1 to 40 mites (average: 12); and we found 1-4 mite species per beetle. Rev. Biol. Trop. 56 (3): 1261-1268. Epub 2008 September 30.
\end{abstract}

Key words: Passalus cognatus, mites, diversity, distribution, Mexico.

Los coleópteros pasálidos son organismos saproxilófagos que viven en túneles dentro de troncos en descomposición, donde realizan todo su ciclo de vida (Reyes-Castillo y Halffter 1984). Los pasálidos presentan hábitos subsociales, ya que durante largo periodo de tiempo forman una estructura social, la cual está determinada por una familia monógama, existiendo cooperación entre el macho y la hembra para el cuidado y alimentación de las crías, así como la cooperación entre individuos hermanos, y el traslape de generaciones (Reyes-Castillo y Halffter 1984). Este comportamiento subsocial parece propiciar su asociación simbiótica con diversos grupos de organismos, como los hongos, los blátidos, los pseudoescorpiones y los ácaros (Reyes-Castillo 2000).

Los pasálidos son organismos característicos de la región intertropical, donde alcanzan su máxima diversidad (Reyes-Castillo 2000). En México la familia Passalidae se distribuye en los ecosistemas forestales húmedos: los bosques mesófilos de montaña y los tropicales perennifolios situados desde el nivel del mar hasta los 3000 metros de altitud (ReyesCastillo 2000).

Se conocen más de 200 especies de ácaros asociados a los pasálidos, que están distribuidas en 68 géneros de 21 familias de los subórdenes Mesostigmata, Prostigmata, Astigmata y Oribatida. Los ácaros de 19 de estas familias se asocian a estos foréticamente (Hunter 1993).

Los ácaros de los pasálidos pueden fijarse al cuerpo de éstos de dos formas generales: 1) sobre el cuerpo en la superficie externa como es en la región gular, en la región frontal de la cabeza, en los alrededores de las coxas, y entre el pro y mesotórax; esto lo realizan principalmente sujetándose a las sedas con los quelíceros o bien empleando las estructuras membranosas (corúnculos) de las patas. 2) los ácaros pueden emplear las cavidades y oquedades del cuerpo 
de los pasálidos para alojarse, como debajo de los élitros, en las esquinas anterolaterales del interior de los élitros, en la región abdominal dorsal del cuerpo, así como en las suturas intersegmentales (Hunter 1993).

Los ácaros han sido recolectados sobre escasas especies de las más de 700 especies descritas de pasálidos, por lo que existen especies de las cuales se desconoce si hospedan poblaciones de ácaros, tal es el caso de Passalus cognatus Truqui. Este pasálido se distribuye en las partes bajas y montañosas de la vertiente del Golfo de México en los estados de Puebla, Veracruz y Oaxaca (Reyes-Castillo y Castillo 1994), donde habita troncos en distintas etapas de descomposición, pertenecientes a numerosas especies arbóreas pioneras o características del bosque maduro; se le encuentra debajo de la corteza, dentro de la zona de alboduramen y en la interfase suelo-tronco podrido (Reyes-Castillo y Castillo 1994). El objetivo del presente trabajo es dar a conocer a los ácaros asociados a Passalus cognatus de Los Tuxtlas, Veracruz.

\section{MATERIALES Y MÉTODOS}

Zona de estudio: Este trabajo se realizó con material recolectado en la Estación Biológica de "Los Tuxtlas", Veracruz, la cual se localiza a $30 \mathrm{~km}$ NE del poblado de Catemaco, en la porción sur del estado de Veracruz $\left(95^{\circ} 04^{\prime}-95^{\circ} 09^{\prime} \mathrm{W}, 18^{\circ} 34^{\prime}-18^{\circ} 36^{\prime}\right.$ Norte), cubre una superficie total de 644 ha. entre un ámbito altitudinal 150 a los $700 \mathrm{~m}$ que se localizan al oriente del Volcán de San Martín Tuxtla, que forman parte de Sierra de los Tuxtlas. Esta última se compone de una sucesión de montañas de orientación diagonal NW-SE, su complejidad geológica, histórica, ecológica y de actividad humana determina la conjunción de una diversidad de ambientes sobre los que se asienta una notable diversidad de especies, tanto de plantas como de animales (Dirzo et al. 1997), como son los pasálidos y sus ácaros asociados.

Obtención de los ácaros: Los ácaros fueron recuperados de pasálidos recolectados durante los años 1985 (enero, febrero junio y diciembre), 1986 (abril y junio), 1996 (octubre) y 2005 (agosto); están depositados en la Colección Entomológica del Instituto de Ecología de Xalapa (CEXA). Los pasálidos se encontraban individualizados en viales de diferentes tamaños con alcohol al 80\%, lo cual nos asegura que los ácaros presentes en el alcohol provienen del ejemplar que se encuentra en el mismo, pero sin saber en que parte del cuerpo se encontraban los ácaros al momento que fue recolectado el pasálido. Los viales estaban en frascos de 2 a 3 litros de capacidad también con alcohol al $80 \%$. Se revisaron un total de 35 individuos de los cuales 20 presentaron ácaros. La revisión se hizo empleando un microscopio estereoscópico y pinzas de punta fina; los ácaros se buscaron en todo el cuerpo, así como en el alcohol donde se encontraban los pasálidos.

Procesamiento del material: Los ácaros se colocaron en frascos de $2 \mathrm{ml}$ y se transportaron a la Colección Nacional de Ácaros (CNAC) donde fueron tratados en una solución de lactofenol por 24 a $72 \mathrm{hr}$ para transparentarlos, se montaron entre porta y cubre objetos con líquido de Hoyer y las preparaciones se colocaron en una estufa a $33^{\circ} \mathrm{C}$ para ser secadas. Los ácaros están depositados en la Colección Nacional de Ácaros en el Instituto de Biología, Universidad Nacional Autónoma de México. Los ejemplares fueron determinados usando los claves de Hunter (1993), Schuster y Summers (1978) y Hunter y Rosario (1991). Los pasálidos fueron determinados usando las claves de Reyes-Castillo (2004).

Análisis estadísticos: A los datos de distribución de los ácaros en las estaciones del año se les realizó prueba de G (The log-like lihood ratio for contigency tables) (Zar, 1974) y la prueba de Chi-cuadrada para determinar si hay relación entre las estaciones del año y el número de pasálidos infestados, considerando al total de pasálidos revisados. Esta última prueba se uso para analizar si hay alguna diferencia en el número de ácaros encontrados sobre los pasálidos infestados durante 
las estaciones del año, usando el programa Statistica (Stat-soft 1995).

\section{RESULTADOS}

Se revisaron 35 ejemplares de Passalus cognatus de los cuales 20 estaban infestados de ácaros y 15 sin ácaros (Cuadro 1), el mayor número de ejemplares se colectó en el verano y el menor en primavera y otoño.

Se recuperaron un total de 245 ácaros: 38 hipopodios, 3 protoninfas, 135 deutoninfas, 16 machos y 53 hembras pertenecientes a ocho géneros, ocho familias y tres subórdenes (Cuadro 2).

Los ácaros más abundantes fueron Uroobovella californiana Wisniewski y
Hirschmann (35\%), Euzercon hyatti Hunter y Rosario (20\%) y Uropoda sp. (17.5\%). Las dos primeras especies se encontraron en un mayor número de pasálidos, con 13 y 9 huéspedes respectivamente (Cuadro 2).

Se obtuvieron ácaros de diferentes estadios de desarrollo (Cuadro 2). De la especie Tenuiplanta crossi (Hunter y Glover) se obtuvieron protoninfas, deutoninfas y adultos (hembras), en el caso de Uroobovella californiana, Uropoda sp. y Mesoplophora sp. solamente se obtuvieron deutoninfas; mientras que de Sancassania sp. únicamente se encontraron hipopodios.

En varios pasálidos se encontraron más de una especie de ácaro, tres ejemplares presentaron cuatro especies y en seis se encontraron tres

CUADRO 1

Ejemplares de Passalus cognatus revisados de Los Tuxtlas, Veracruz, México, según la estación

TABLE 1

Specimens of Passalus cognatus examined from Los Tuxtlas, Veracruz, Mexico, per season

\begin{tabular}{|c|c|c|c|c|}
\hline Pasálidos & Primavera & Verano & Otoño & Invierno \\
\hline Con ácaros & 4 & 10 & 1 & 5 \\
\hline Sin ácaros & 1 & 5 & 4 & 5 \\
\hline Total & 5 & 15 & 5 & 10 \\
\hline
\end{tabular}

\section{CUADRO 2}

Ácaros asociados a Passalus cognatus de Los Tuxtlas, Veracruz, Mexico TABLE 2

Mites associated to Passalus cognatus from Los Tuxtlas, Veracruz, Mexico

\begin{tabular}{|c|c|c|c|c|}
\hline Subórden & Familia & Especie & Ejemplares & Huéspedes \\
\hline Astigmata & Acaridae & Sancassania sp. & 38 hi & 7 \\
\hline \multirow[t]{6}{*}{ Mesostigmata } & Euzerconidae & Euzercon hyatti & 37 ơ, 12 రొ & 9 \\
\hline & Diarthrophallidae & Tenuiplanta crossi & $3 \stackrel{9}{ } 3,5 \mathrm{D}, 3 \mathrm{P}$ & 4 \\
\hline & Diplogyniidae & Tridiplogynium sp. & 7 ధి, 4 ठ ठ & 4 \\
\hline & Laelapidae & Hypoaspis disjuncta & 6 qQ & 2 \\
\hline & Urodinychidae & Uroobovella californiana & $86 \mathrm{D}$ & 13 \\
\hline & Uropodidae & Uropoda sp. & $43 \mathrm{D}$ & 5 \\
\hline Oribatida & Mesoplophoridae & Mesoplophora sp. & $1 \mathrm{D}$ & 1 \\
\hline
\end{tabular}

hi $=$ Hipopodio, $\mathrm{P}=$ Protoninfa, $\mathrm{D}=$ Deutoninfa. 
especies, la mayoría de los ejemplares con más especies fueron recolectados durante el verano.

Los ácaros se localizaron principalmente, en las coxas de las patas de los pasálidos (49.8\%), seguidos en menor abundancia en los húmeros $(5.3 \%)$, en el mesoesternón (4.08\%) y debajo de las alas membranosas (2.04\%) (Cuadro 3). En las coxas de las patas se encontraron principalmente a las deutoninfas y los hipopodios; mientras que en el mesoesternón, los húmeros y debajo de las alas membranosas se encontraron a los adultos.

Los ácaros también se encontraron en el alcohol donde están almacenados los pasálidos (38.78\%), en cuatro pasálidos se encontraron sobre el cuerpo y en el alcohol y en otros cuatro solamente en el alcohol.

Las especies de ácaros se encontraron en mayor proporción en el verano (ocho especies), seguidas por invierno (cuatro), primavera (tres) y otoño (dos) (Cuadro 4), sólo Uroobovella californiana se encontró en todas las estaciones, mientras que Euzercon hyatti se encontró de verano a invierno y Uropoda sp. sólo se ausentó en el otoño. Tres de las ocho especies únicamente se recolectaron en el verano (Cuadro 3).

\section{DISCUSIÓN}

Se encontraron ocho especies de ácaros sobre el pasálido Passalus cognatus. Este número es cercano a las 11 especies de ácaro registradas sobre el pasálido Odontotaenius disjunctus (Illiger) por Pearse y colaboradores (1936), quienes revisaron 120 ejemplares durante un ciclo anual de junio de 1933 a mayo de 1934. Los ejemplares de P. cognatus presentaron de uno a cuarenta ácaros, el promedio fue de doce. Este promedio es mayor al registrado por Hunter y Mollin (1964), quienes examinaron 999 ejemplares de Odontotaenius disjunctus y encontraron 2.8 ácaros por coleóptero.

Al comparar nuestros resultados con los publicados por Hunter y Davis (1965) quienes reportan que de 1142 ejemplares examinados el $38 \%$ presentaron ácaros con un promedio de 2.6 ácaros por coleóptero, obtuvimos un porcentaje mayor en este trabajo con el $52 \%$ del total de los organismos revisados con ácaros y un promedio ácaro/pasálido cuatro veces mayor.

El mayor número de ácaros encontrado sobre un ejemplar de P. cognatus fue de 40, número significativamente menor comparado

CUADRO 3

Ubicación de los ácaros sobre Passalus cognatus de Los Tuxtlas, Veracruz, México

TABLE 3

Location of the mites on Passalus cognatus from Los Tuxtlas, Veracruz, Mexico

\begin{tabular}{|c|c|c|c|c|c|}
\hline Especie & Coxas & Húmeros & Mesosternón & Alas* & Alcohol \\
\hline Sancassania sp. & 35 & & & & 3 \\
\hline Euzercon hyatti & 12 & 8 & 5 & & 24 \\
\hline Tenuiplanta crossi & 4 & & & & 7 \\
\hline Uroobovella californiana & 64 & 5 & 5 & & 12 \\
\hline Uropoda sp. & 1 & & & & 42 \\
\hline Hypoaspis disjuncta & 1 & & & 5 & \\
\hline Tridiplogynium sp. & 5 & & & & 6 \\
\hline Mesoplophora sp. & & & & & 1 \\
\hline Total & 122 & 13 & 10 & 5 & 95 \\
\hline
\end{tabular}


CUADRO 4

Distribución estacional de los ácaros sobre Passalus cognatus de Los Tuxtlas, Veracruz, México

TABLE 4

Seasonal distribution of the mites on Passalus cognatus from Los Tuxtlas, Veracruz, Mexico

\begin{tabular}{|c|c|c|c|c|}
\hline Especies & Primavera $(n=4)$ & Verano $(n=10)$ & Otoño $(\mathrm{n}=1)$ & Invierno $(\mathrm{n}=5)$ \\
\hline Sancassania sp. & 6 & 32 & & \\
\hline Euzercon hyatti & & 34 & 8 & 7 \\
\hline Tenuiplanta crossi & & 11 & & \\
\hline Uroobovella californiana & 41 & 29 & 10 & 6 \\
\hline Uropoda sp. & 4 & 35 & & 4 \\
\hline Hypoaspis disjuncta & & 1 & & 5 \\
\hline Tridiplogynium sp. & & 11 & & \\
\hline Mesoplophora sp. & & 1 & & \\
\hline Total & 51 & 154 & 18 & 22 \\
\hline
\end{tabular}

con los 189 urópodos encontrados por Gordh y Willis (1989) sobre un ejemplar del tenebriónido Alphitobius diaperinus (Panzer).

Con respecto al número de especies de ácaros por ejemplar de pasálido éste varía de uno a cuatro especies. En tres de los pasálidos se encontraron cuatro especies de ácaros, de los cuales comparten dos Euzercon hyatti y Uroobovella californiana, sin embargo, al comparar por pares de ejemplares comparten hasta tres especies. Esta similitud en las especies registradas sobre cada pasálido, probablemente se deba a que fueron recolectados en el mismo día y lugar (4 de junio, 1986), desconocemos si estaban en el mismo tronco, ya que de ser así es coherente que compartan las mismas especies porque estaban compartiendo el mismo hábitat. Este dato es importante que se considere para futuras colectas, porque así podremos explicar la distribución y relación entre los ácaros de los pasálidos.

De los ácaros recolectados se encontraron distintos estadios de desarrollo, en el caso de Tenuiplanta crossi se encontraron tres de los estadios de desarrollo (Cuadro 2) en un mismo organismo, lo cual sugiere que esta especie realiza todo su ciclo de vida sobre este pasálido o en su madriguera. En algunas especies sólo se encontró un estadio, como es el caso de Sancassania sp., de la cual sólo se obtuvieron hipopodios, que son estadios deuteroninfales especializados, resistentes a la desecación y a otras condiciones ambientales adversas, carecen de partes bucales funcionales y gnatosoma, este estadio es la fase dispersora del ciclo de vida de algunos ácaros de vida libre que presenta adaptaciones morfológicas para realizar la foresia (Evans 1992).

En el caso de Euzercon hyatti de la que sólo se encontraron adultos es posible que este sea el único estadio que realiza la foresia. Los estadios ninfales, podrían encontrarse en la hojarasca y suelo debajo de los troncos en descomposición, como habita con E. latus (Banks) (Hunter y Davis 1965).

Para las especies U. californiana y Uropoda sp. de las que sólo se encontraron deutoninfas, esto se debe a que en este estadio presenta glándulas anales las cuales secretan una sustancia que al secarse forma un pedicelo o uropodo que le sirve para fijarse a la superficie del huésped y realizar la foresia (Athias-Binche 1994). 
La presencia de Mesoplophora sp. puede ser considerada accidental, ya que esta especie es propia del suelo y posiblemente fue recolectado con fragmentos de suelo al momento de recolectar al pasálido, esto se corrobora ya que el organismo se encontró en el alcohol y no sobre el cuerpo del coleóptero, sin embargo este género se ha encontrado forético en dictiópteros (Evans 1992).

Las zonas del cuerpo donde más se encontraron a los ácaros fueron en las coxas I, II y III, lo que coincide con lo registrado por Hunter y Davis (1965) quienes encontraron que el ácaro Euzercon latus (Banks) prefiere fijarse en los alrededores de las coxas I y II sobre Odontotaenius disjunctus (Illiger). En estas áreas se presentaron principalmente las deutoninfas, protoninfas e hipopodios; esto puede deberse a que aquí encuentran pequeñas cavidades donde se introducen, en el caso de hipopodios y protoninfas, para protegerse de las condiciones ambientales y a las deutoninfas les permiten fijarse más fácilmente al cuerpo del coleóptero. Hypoaspis disjuncta se ubicó en las alas membranosas y coxas (Cuadro 3), lo cual coincide parcialmente con los registros previos ya que se habían encontrado en la parte ventral del protórax, en las partes bucales y en las coxas (Hunter y Yeh 1969, Delfinado y Baker 1975).

En el mesoesternón y en los húmeros se encontraron comúnmente adultos, los que se sujetan a las sedas de estas estructuras y generalmente se encuentran varios individuos juntos. Algunos se encontraron en el alcohol donde se preservaba al pasálido, ya que al capturar al organismo y colocarlo en el vial, el ácaro que no estaba bien sujeto al huésped se desprendió. Las deutoninfas son abundantes en el alcohol, esto puede deberse a que al contacto con el alcohol el pedicelo que utilizan para fijarse se disuelva o que el ácaro se desprenda del pedicelo.

Si bien se ha observado que los hipopodios suelen ubicarse debajo de los élitros y las alas membranosas (Athias-Binche 1994), no se encontró ningún ejemplar en estas condiciones. Esto posiblemente se deba a que los pasálidos se recolectaron dentro de troncos, donde las condiciones ambientales, humedad y temperatura, son óptimas y los hipopodios no tienen que resguardarse de la desecación.

Se aplicó a los datos de pasálidos con o sin ácaros de las cuatro estaciones del año (Cuadro1) la prueba de $\mathrm{G}$ y la de chi-cuadrada $\left(\mathrm{G}=4.8 ; \chi^{2}=7.815, \mathrm{p}<0.05\right)$, los resultados muestran que no existe relación entre las estaciones del año y la presencia/ausencia de ácaros; puesto que el valor de $\mathrm{G}$ es menor a la de chi-cuadrada.

Los ácaros sobre los pasálidos fueron más abundantes en el verano con 154 organismos (Cuadro 4), disminuyendo la abundancia en primavera 51, en el invierno 22 y en el otoño 18. A estos resultados se les aplicó una prueba chi-cuadrada y no se encontraron diferencias significativas $\left(\chi^{2}=197.85, \mathrm{df}=3, \mathrm{P}<0.0000\right)$. Estos resultados coinciden con lo registrado por Hunter y Davis (1965) quienes encontraron que en junio, julio y agosto el número de ácaros sobre $O$ disjunctus es mayor.

Los datos encontrados de la distribución de los ácaros en las diferentes estaciones del año probablemente están asociados al de organismos recolectados y revisados, ya que fueron pocos comparados con otros trabajos similares donde se han revisado más de 900 ejemplares (Hunter y Mollin 1964), y la continuidad en el ciclo anual. Por lo cual la distribución encontrada sólo pude considerarse como una pequeña muestra de los ácaros que pueden estar asociados con Passalus cognatus y se requiere de un periodo de recolecta anual completo para determinar la variación estacional de los ácaros en esta especie. Sin embargo, nuestros resultados (Cuadro 4) coinciden con lo registrado por Pearse et al. (1936), quienes encontraron al género Uroobovella en todas las estaciones del año sobre $O$. disjunctus, por lo cual, lo consideran como un grupo de ácaros erráticos.

Debido al número de especies y ejemplares de ácaros que se encuentran sobre los pasálidos estos deben ser considerados una comunidad, en la cual seguramente hay interacciones entre sus integrantes, pero la información que se conoce sobre la biología y ecología de muchas 
de estas especies es escasa por lo que se les considera como simples organismos foréticos. Sin embargo, se sabe que los ácaros trigináspidos, a los que pertenecen los euzercónidos, son organismos generalistas y en ocasiones juegan el rol de depredador, de fungívoro o de carroñero (Seeman y Walter 1997), un ejemplo de ello son los ejemplares adultos de Euzercon latus que se alimentan de huevos de colémbolos, colémbolos dañados y coleópteros recién muertos, mientras que los inmaduros son carroñeros y fungívoros (Hunter y Davis 1965). Por lo cual es posible que se realice algún tipo de simbiosis entre los ácaros foréticos. En nuestros resultados encontramos que en los pasálidos donde si había ejemplares de $E$. hyatti el número de hipopodios de Sancassania sp. era escaso, de cero a tres, y cuando $E$. hyatti está ausente el número de hipopodios era abundante (22), por lo que es probable que los euzercónidos encontrados se estén alimentando de los hipopodios. Hay registros de ácaros que depredan a otros como es el caso de Macrocheles muscadomesticae (Scopoli) que se alimenta de Caloglyphus spp. (Axtel 1967), por lo cual la idea arriba plantada merece ser analizada rigurosamente.

En el caso de los géneros Uropoda y Uroobovella se tienen registros de que depredan a nemátodos e hifas de hongos (Evans 1992), respectivamente, por lo que es probable que en los troncos en descomposición encuentren su alimento y efectivamente su relación con los pasálidos sea meramente forética.

Los resultados nos dan una idea de la diversidad de organismos que se encuentran asociados con los pasálidos. Esto posiblemente se debe al tipo de hábitat donde los coleópteros se desarrollan. Los troncos en descomposición proporcionan un ambiente relativamente estable, donde parece existir una escasa variación de temperatura y humedad, propiciando el establecimiento de una gran variedad de organismos a lo largo de su degradación (Castillo y Reyes-Castillo 2003). Los ácaros viven en los túneles que construyen los pasálidos (Hunter 1993) y es ahí donde se suben a estos para ser transportados a nuevos túneles o troncos. Los pasálidos juegan un papel importante en la dis- tribución de algunos ácaros. Un ejemplo de ello son los urópodos, que sólo son foréticos en su etapa de deutoninfa y después de ser transportados a otro hábitat donde encuentren mejores condiciones para continuar con su desarrollo, se benefician por la reducción de competencia por el hábitat y de prevenir la endogamia.

Consideramos que la mayoría de los ácaros asociados a Passalus cognatus pueden ser considerados foréticos, sin embargo, se deben hacer estudios más precisos para determinar si esta es la razón por la que los ácaros se suben al coleóptero y para estudiar las interacciones entre las especies de ácaros que habitan en los túneles de este pasálido.

\section{AGRADECIMIENTOS}

Los autores agradecen a Oscar Francke, Oscar J. Polaco, Cristina Kramer y Mario Vargas por sus comentarios y sugerencias al manuscrito, así como a Ma Luisa Castillo por la recolecta de los pasálidos y a Enrique Montes de Oca su ayuda en la prueba estadística. Este estudio fue apoyado por el CONACYT y el Posgrado en Ciencias Biológicas de la UNAM.

\section{RESUMEN}

Se revisaron 35 ejemplares de Passalus cognatus Truqui recolectados en Los Tuxtlas, Veracruz, México. En 20 de ellos se encontraron 245 ácaros pertenecientes a ocho especies, ocho géneros, ocho familias y tres subórdenes. Las especies de acáros más abundantes fueron Uroobovella californiana Wisniewski y Hirschmann (35\%), Euzercon hyatti Hunter y Rosario (20\%) y Uropoda sp. (17.5\%). Las zonas de fijación preferidas por los ácaros fueron las coxas; en menor proporción el mesoesternón y los húmeros. Los pasálidos infestados presentaron de 1 a 40 ácaros, con un promedio de 12. El número de especies de ácaros por pasálido infestado fue de 1 a 4 .

Palabras Clave: Passalus cognatus, ácaros, diversidad, distribución, México

\section{REFERENCIAS}

Athias-Binche, F. 1994. La phorésie chez les acariens. Aspects adaptatifs et Evolutifs. Castillet, Perpignan, París, Francia. 178 pp. 
Axtel, R.C. 1967. Macrochelidae (Acarina: Mesostigmata) as biological control agents for synantropic flies, p. 401-416. In G. O. Evans (ed.). Proceedings of the 2nd Internacional Congress of Acarology. Akadémiai Kiadó, Budapest, Hungría.

Castillo, M.L. \& P. Reyes-Castillo. 2003. Los Passalidae: coleópteros tropicales degradadores de troncos de árboles muertos, p. 237-262. In J. Álvarez-Sánchez y E. Naranjo-García (eds.). Ecología del suelo de la selva tropical húmeda de México. Instituto de Ecología, A. C., Instituto de Biología y Facultad de Ciencias UNAM, Xalapa, Veracruz, México.

Delfinado, M.D. \& E.W. Baker. 1975. Mites (Acarina) associated with Popilius disjunctus (Illiger) (Coleoptera: Passalidae) in eastern United States. J.N.Y. Entomol. Soc. 83: 49-59.

Dirzo, R., E. González Soriano \& R.C. Vogt. 1997. Introducción general, p. 3-6. In R. Dirzo, E. González Soriano y R. C. Vogt (eds.) Historia Natural de los Tuxtlas. Universidad Nacional Autónoma de México, Ciudad de México, Distrito Federal, México.

Evans, G.O. 1992. Principles of acarology. CAB International, Oxon, Inglaterra 563 pp.

Gordh, G. \& L. Wills. 1989. Anatomical notes on Uropoda sp., a phoretic mite infesting dung-inhabiting beetles in Southern California (Acari: Uropodidae; Coleoptera: Tenebrionidae, Histeridae). Pan-Pac. Entomol. 65: 410-413.

Hunter, P.E. 1993. Mites associated with New World passalid beetles (Coleoptera: Passalidae). Acta Zool. Mex. Nueva Ser. $58: 1-37$.

Hunter, P.E. \& R. Davis. 1965. Mites associated with the passalus beetle III. Life stages and observations on biology of Euzercon latus (Banks) (Acarina: Euzerconidae). Acarologia. 6: 247-256.

Hunter, P.E. \& K. Mollin. 1964. Mites associated with the passalus beetle I. Life stages and seasonal abundance of Cosmolaelaps passali $\mathrm{n}$. sp. (Acarina: Laelapidae). Acarologia. 6: 247-256.
Hunter, P.E. \& S.M. Yeh. 1969. Hypoaspis (Geolaelaps) disjuncta, n. sp. (Acarina: Laelapidae) associated with the horned passalus beetles. J. Ga. Entomol. Soc. 4:97-102.

Hunter, P.E. \& R. M. T. Rosario. 1991. New species of Euzercon from Mexico with a key to the new world species (Acarina: Mesostigmata: Euzerconidae). Acarologia. 32:205-216.

Pearse, A.S., M.T. Patterson, J.S. Rankin \& G.W. Wharton. 1936. The ecology of Passalus cornutus Fabricius, a beetle which lives in rotting logs. Ecol. Monogr. 6:455-490.

Reyes-Castillo, P. 2000. Coleoptera Passalidae de México, p. 171-182. In F. Martín-Piera, J. J. Morrone \& A. Melic (Eds.). Hacia un proyecto Cyted para el inventario y estimación de la diversidad entomológica en Iberoamérica: Pribes 2000, M3m: Monografías Tercer milenio, Sociedad Entomológica Aragonesa, Zaragoza, España.

Reyes-Castillo, P. 2004. La tribu Passalini (Coleoptera: Passalidae, Passalinae) en México. Tesis de Doctorado en Ciencias Biológicas, Universidad Autónoma Metropolitana. México, D. F. 170 pp.

Reyes-Castillo, P. \& M.L. Castillo. 1994. Revalidación de Passalus (Pertinax) cognatus Truqui (Coleoptera: Passalidae). Folia Entomol. Mex. 92:75-76.

Reyes-Castillo, P. \& G. Halffter. 1984. Estructura social de los Passalidae (Coleoptera: Lamellicornia). Folia Entomol. Mex. 61:49-72.

Schuster, R.O. \& F.M. Summers. 1978. Mites of the family Diarthrophallidae (Acari: Mesostigmata). Int. J. Acarol. 4:279-385.

Seeman O.D. \& D.E. Walter. 1997. A new species of Triplogyniidae (Mesostigmata: Celaenopsoidea) from Australian rainforests. Int. J. Acarol. 23:49-59.

StatSoft, Inc. 1995. Statistica for Windows. StatSoft, Tulsa, Oklahoma, EEUU.

Zar, J. H. 1974. Biostatistical Analysis. Prentince Hall, Inc. A Simon \& Scuster Company. Englewood Cliffe, Nueva Jersey, EEUU. 